\title{
Microgrid Security Assessment and Islanding Control by Support Vector Machine
}

\author{
Yongli Zhu, Riyasat Azim, Hira Amna Saleem, Kai Sun \\ Department of Electrical Engineering \\ University of Tennessee \\ Knoxville, USA \\ yzhu16@vols.utk.edu, mazim@vols.utk.edu, \\ hiraamna@hotmail.com, kaisun@utk.edu
}

\begin{abstract}
A Support Vector Machine (SVM) based approach for microgrid islanding decision and control is investigated. The IEEE 13-feeder system is modified and serves as the microgrid model connected to Kundur four-machine two-area system that models the main transmission grid. A representative data set is obtained through simulations in MATLAB/Simulink considering multiple typical scenarios with or without a fault. A SVM classifier is designed to identify insecure scenarios with satisfying accuracy. Comparisons between different kernel functions are then carried out, which indicate that linear SVM can be effective for the islanding control. The SVM approach is further compared with a decision tree based approach in terms of training and testing accuracies for the microgrid islanding control problem.
\end{abstract}

Index Terms-Decision tree; islanding; machine learning; microgrid; SVM

\section{INTRODUCTION}

As a promising technique for more efficient utilization of renewable resources, microgrid technology has gained more and more attention; its advantages include more flexible control schemes for local sources and the ability to operate in the islanded mode. Distributed generators (DG) like photovoltaics (PV) and fuel cells, are usually small units compared to conventional generations, most of which are equipped with power electronic $(\mathrm{PE})$ interface. With diverse DGs incorporated and interactions with the main grid, operations with a microgrid are different from transmission grid operations. To ensure the safety of microgrid operations, security assessment (SA) needs to be done, where a key issue is how to design an effective islanding control scheme involving the microgrid and main grid. The main challenges include: 1) variable short-circuit current level due to PE converter capacity limits and renewable fluctuations 2) transitioning between two operation modes, i.e. the grid-tied mode and islanded mode.

Some related efforts have considered physical properties of DG sources so as to design logically complicate protection schemes or islanding detection algorithms implemented with differential relays [1] and DG local measuring equipment [2]. On the other hand, traditional SA involves numerical solution of multiple high-order nonlinear differential algebraic

\author{
Di Shi, Ratnesh Sharma \\ Department of Energy management \\ NEC Laboratories America, Inc. \\ Cupertino, USA \\ dshi@nec-labs.com \\ ratnesh@nec-labs.com
}

equations (DAEs) and is computationally inefficient for online purposes. Therefore, machine learning techniques such as Decision Tree (DT), Support vector machine (SVM), AdaBoost, etc. provide alternative insight for SA problems [3-5]. The basic idea is to train a classifier offline and use it for online SA; when an insecure condition is identified, a preventive control action will be taken to bring the system back to safe region such as islanding the microgrid from the main grid or adjusting DGs' outputs. Among these machine learning methods, the DT has been widely investigated for applications in power system SA due to its simplicity and white-box nature as compared to blackbox machine learning techniques [3]-[7]. For the SVM, its multi-class versions have been successfully tested on IEEE 39 and 118 bus systems [8]. Applications of the SVM for post-fault stability prediction using PMU data are presented in [9] and [10]. The SVM can also be adopted to improve settings of distance relay by discerning three different types of events: fault, power swing, and voltage instability [11]. For a microgrid, the SVM is a promising method for SA and islanding decision purpose, since the aforementioned studies have demonstrated its good performance on small-scale classification problems.

This paper focuses on islanding and decision and control for a microgrid integrating different types of DGs, i.e. a diesel generator, a battery and a photovoltaic generator $(\mathrm{P})$. This paper is organized as follows: in section II, the microgrid model is introduced and its islanding decision problem is described; in section III, the basic principle of SVM is introduced, and the dataset generation and SVM training are discussed in detail. Section IV compares three different kernels. Numerical results are presented in section IV while section V concludes the whole paper.

\section{FUNDAMENTALS OF THE SVM}

\section{A. Basic idea}

The SVM is a kind of large margin classifiers. Its decision function is determined by a subset of training samples, i.e., the so-called "support vectors". Its solution process involves quadratic programming techniques. Compared with traditional classifiers like DT and ANN, the advantages of SVM include: 
1) Clear and strict mathematic foundation, i.e. maximize the distance between decision surface and boundary points (i.e. support vectors) of different classes.

2) Higher accuracy for small sample volume learning.

3) Multiple choices of different nonlinear kernel functions and theoretically proofed generalization ability. Its basic idea is shown in Fig. 3.

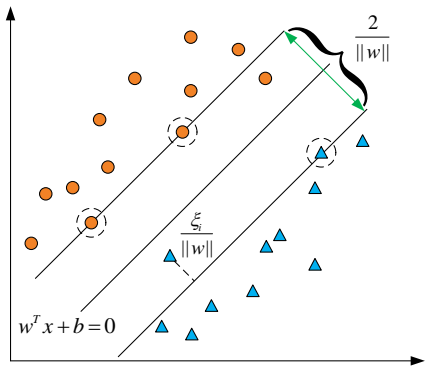

Figure 1. Basic idea of SVM classifier

\section{B. Soft margin SVM}

Even for a linear SVM, typically most cases are not linearly separable. Thus, slack variable $\xi_{i}$ is introduced to "loosen" the original constraints. Meanwhile, a cost parameter $C$ is introduced to penalize the misclassified points (a larger $C$ implies a higher penalty). Thus, the mathematic model of SVM becomes the following convex quadratic programming problem (primal form):

$$
\begin{array}{ll}
\min _{w, b, \xi} & \frac{1}{2}\|w\|^{2}+C \sum_{i=1}^{N} \xi_{i} \\
\text { s.t. } & y_{i}\left(w \cdot x_{i}+b\right) \geq 1-\xi_{i}, \quad \\
& \xi_{i} \geq 0, \quad i=1,2, \ldots N
\end{array}
$$

Solving this problem involves Lagrange dual technique from optimization theory [13].

\section{Nonlinear SVM and Kernel trick}

In most cases, the dataset cannot be ideally separated by linear SVM. This is solvable by mapping the feature space into another dimension space as shown in Fig. 4 through some nonlinear mapping relation (2).

$$
\Phi: x \rightarrow \phi(x)
$$

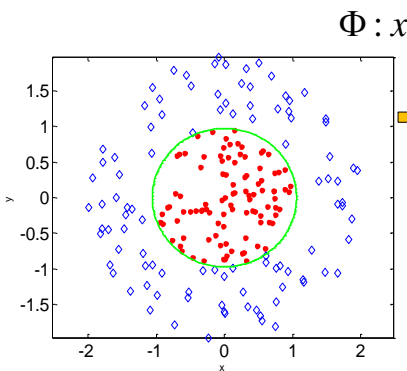

$$
\Phi: x \rightarrow \phi(x)
$$

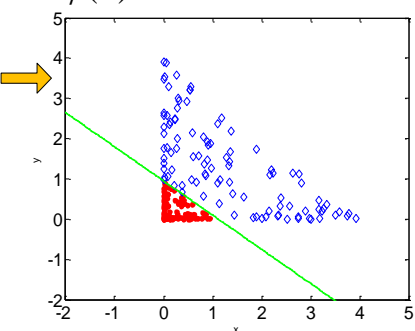

Figure 2. Nonlinear mapping of feature space by kernel function
The "Kernel Trick": in practical applications, most problems are not linearly classifiable, thus SVM adopts a "kernel" function $K\left(x_{i}, x_{j}\right)$ to map the data into a new space. The merit is that no explicit form of the map $\phi(x)$, but only replacing the inner product $\left\langle x_{i}, x_{j}\right\rangle$ by the kernels is needed. Some commonly used kernel functions are:

- Polynomial kernel function

$K(x, z)=(1+x z)^{p}$

This is a polynomial of degree $p$. The decision function is:

$$
f(x)=\operatorname{sign}\left(\sum_{i=1}^{N} a_{i}^{*} y_{i}\left(x_{i} \cdot x+1\right)^{p}+b^{*}\right)
$$

- Gaussian kernel function

$$
K(x, z)=\exp \left(-\frac{\|x-z\|^{2}}{2 \sigma^{2}}\right)
$$

This formula is also known as radial basis function or RBF kernel in brief. Its decision function has the following form:

$$
f(x)=\operatorname{sign}\left(\sum_{i=1}^{N} a_{i}^{*} y_{i} \exp \left(-\frac{\|x-z\|^{2}}{2 \sigma^{2}}\right)+b^{*}\right)
$$

By the "Kernel Trick", a SVM can be applied to classification problems that are linearly inseparable in the original space.

III. MICROGRID ISLANDING DECISION AND CONTROL

\section{A. Microgrid modeling}

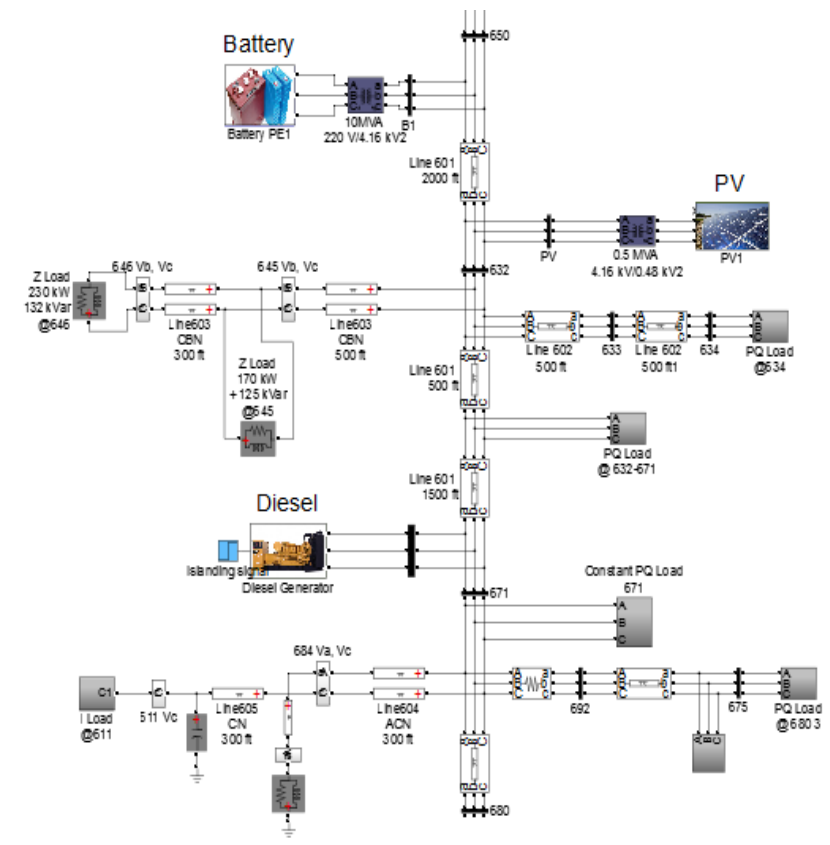

Figure 3. Microgrid model: IEEE 13 feeder system

As shown by Fig. 3, the microgrid was modeled in MATLAB/Simulink as the data generating platform. The IEEE 13 feeder system is modified and serves as the microgrid model in this paper. Three DGs, i.e. a battery, a Photovoltaic (PV) 
panel, and a diesel generator are added to bus 650, 632 and 671 of the original system [12]. The total load demand is $2.69 \mathrm{MW}$ for active power and 1.9 MVar for reactive power.

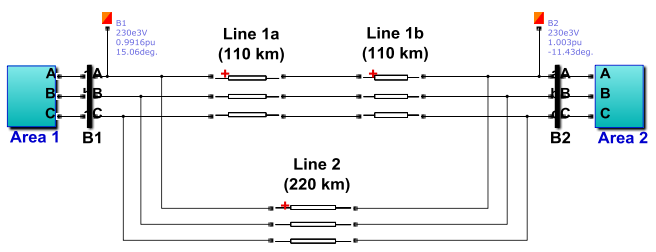

Figure 4. Main grid model: Kundur's two area system

The main grid is Kundur's two area system as showed in Fig. 3. The microgrid is connected in area 2. The presented SVM based islanding control method is at the utility side, which means only measurements at the PCC (point of common coupling) bus are needed. Its goal is to identify insecure operating conditions for microgrid in a fast and reliable way.

\section{B. Dataset generation}

In order to enrich the database of scenarios, five microgrid loading levels are designed: $50 \%, 75 \%, 100 \%, 125 \%$ and $150 \%$ of its nominal value. Detailed scenario descriptions are as follows:

- DG availability interruption: consider 7 types of scenarios with some DGs becoming unavailable due to maintenance or other unplanned reasons: one DG is out (3 cases), two DGs are out (3 cases), and all DGs are unavailable (1 case). Thus $7 \times 5=35$ cases are created for 5 loading levels.

- Microgrid load changes: microgrid load is increased by $25 \%, 50 \%$ and decreased by $25 \%, 50 \%$ at 2 s for each of 5 loading levels, then after 1 cycle, returns to the original value. Thus, totally $4 \times 5=20$ cases are created.

- Main grid load changes: load decreases by $100 \%, 50 \%$ at $2 \mathrm{~s}$ respectively in Area-1 and Area-2 for 5 microgrid loading levels, then after one cycle, returns to the original value. Thus totally $4 \times 5=20$ cases are created.

- Battery output variation: the battery decreases its active power from the original 1.0pu o $0.5 \mathrm{pu}$ for each load level.

- Fault events: consider a fault occurs at each of 6 selected buses, i.e. two buses in Area-1, two buses in Area-2 and two buses at tie-line terminals. There are 7 fault types: single-phase ground (3), phase-to-phase (3) and 3-phase ground (1). Thus, totally $6 \times 7 \times 5=210$ cases are created.

- Original normal case (without any event): totally 5 cases for 5 loading levels.

Finally, the total data set has 210 insecure cases and 85 secure cases.

\section{SVM classifier design}

The SVM is used to extract high-level knowledge from the generated dataset. The SVM tools used are LIBSVM [13] and Weka [14]. Each case the dataset is characterized by a set of attributes from system measurements, which are used as inputs to the SVM. The 16 attributes used are listed in Table 1. The values of attributes are obtained from measurements at a sampling rate of $60 \mathrm{~Hz}$, which is chosen based on two considerations: first, the industry practice is above $25 \mathrm{~Hz}$, typically $30 \mathrm{~Hz}$ or $60 \mathrm{~Hz}$; second, $60 \mathrm{~Hz}$ is preferable since a higher sampling rate theoretically means a higher quality of inputs for the SVM classifier. The voltage and frequency security criteria and classification tags for the simulation results are listed in Table 2 and Table 3, respectively.

TABLE I. ORIGINAL ATtRIBUtes LIST FOR SVM

\begin{tabular}{|l|l|}
\hline \multicolumn{1}{|c|}{ Attribute name } & \multicolumn{1}{c|}{ Physical Meaning } \\
\hline Vamin & Minimum voltage at PCC bus phase-A \\
\hline Vamax & Maximum voltage at PCC bus phase-A \\
\hline Vbmin & Minimum voltage at PCC bus phase-B \\
\hline Vbmax & Maximum voltage at PCC bus phase-B \\
\hline Vcmin & Minimum voltage at PCC bus phase-C \\
\hline Vcmax & Maximum voltage at PCC bus phase-C \\
\hline Iamin & Minimum current at PCC bus phase-A \\
\hline Iamax & Maximum current at PCC bus phase-A \\
\hline Ibmin & Minimum current at PCC bus phase-B \\
\hline Ibmax & Maximum current at PCC bus phase-B \\
\hline Icmin & Minimum current at PCC bus phase-C \\
\hline Icmax & Maximum current at PCC bus phase-C \\
\hline Pmin & Minimum active power at PCC bus \\
\hline Pmax & Maximum active power at PCC bus \\
\hline Qmin & Minimum reactive power at PCC bus \\
\hline Qmax & Maximum reactive power at PCC bus \\
\hline
\end{tabular}

TABLE II. Voltage Event ClasSificAtion CRITERIA

\begin{tabular}{|l|l|}
\hline Under-Voltage 2 (VU2) & $\mathrm{V}<0.5 \mathrm{pu}$ \\
\hline Under-Voltage 1 (VU1) & $0.5 \mathrm{pu} \leqslant \mathrm{V}<0.88 \mathrm{pu}$ \\
\hline Normal-Voltage (VN) & $0.88 \mathrm{pu} \leqslant \mathrm{V}<1.1 \mathrm{pu}$ \\
\hline Over-Voltage 1 (VO1) & $1.1 \mathrm{pu} \leqslant \mathrm{V}<1.37 \mathrm{pu}$ \\
\hline Over-Voltage 2 (VO2) & $\mathrm{V} \geqslant 1.37 \mathrm{pu}$ \\
\hline
\end{tabular}

TABLE III. FREQUENCY EVENT ClasSIFICATION CRITERIA

\begin{tabular}{|l|l|}
\hline Under-Frequency 2 (FU2) & $\mathrm{f}<57 \mathrm{~Hz}$ \\
\hline Under-Frequency 1 (FU1) & $57.0 \mathrm{~Hz} \leqslant \mathrm{f}<59.8 \mathrm{~Hz}$ \\
\hline Normal Frequency (FN) & $59.8 \mathrm{~Hz} \leqslant \mathrm{f}<60.5 \mathrm{~Hz}$ \\
\hline Over-Frequency 1 (FO1) & $\mathrm{f} \geqslant 60.5 \mathrm{~Hz}$ \\
\hline
\end{tabular}

\section{RESULTS AND ANALYSIS}

In the following study, class " 1 " means "secure" while class " 0 " means "insecure", i.e. islanding decision to be made.

\section{A. Trainning results using all the attributes}

\section{1) Without SVM parameter optimization}

TABLE IV. Results Using All The Attributes

\begin{tabular}{|l|l|}
\hline Correctness & $98.9831 \%(292 / 295)$ \\
\hline kernel type & RBF kernel \\
\hline gamma (i.e. $\gamma)$ & 0.0078125 \\
\hline total support vector number. & 35 \\
\hline label & 1 : insecure 0: secure \\
\hline $\begin{array}{l}\text { nr_sv: number of support } \\
\text { vectors (SVs) for each class }\end{array}$ & $\begin{array}{l}\text { 17 SVs for class "11" } \\
18 \text { SVs for class "0" }\end{array}$ \\
\hline
\end{tabular}

From the table above, we can see there are three misclassified cases (class " 1 " being misclassified as " 0 "). 
Thus, even using default parameters in SVM training, it still achieves a satisfying accuracy $>98 \%$.

\section{2) With SVM parameter optimization}

In the previous training procedure, parameters $C$ and $\gamma$ are not optimized. The LIBSVM toolbox provides a tool for choosing a pair of best parameters through a so-called Grid Search Algorithm [13]. From optimization, $C=8.0$ (default value=1.0) and $\gamma$ remains 0.0078125 . The search process is shown in Fig. 5. The new result is shown in Table 5.

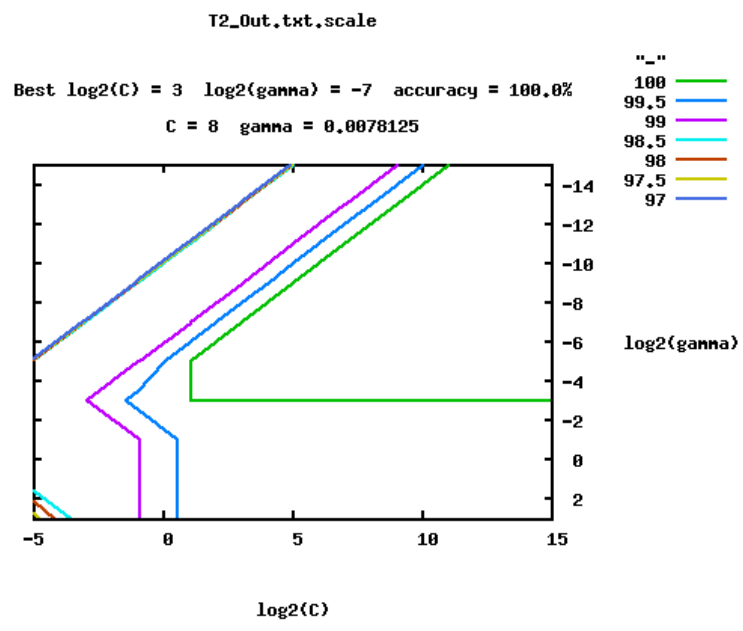

Figure 5. Grid search process for SVM parameter optimization

TABLE V. RESULTS USING OPTIMIZAED PARAMETERS

\begin{tabular}{|l|l|}
\hline Correctness & $100 \%(295 / 295)$ \\
\hline kernel type & RBF kernel \\
\hline gamma (i.e. $\gamma$ ) & 0.0078125 \\
\hline total support vector number. & 19 \\
\hline label & 1 insecure 0: secure \\
\hline $\begin{array}{l}\text { nr_sv (number of SV for each } \\
\text { class) }\end{array}$ & $\begin{array}{l}5 \text { sv for class "1" } \\
14 \text { sv for class "0" }\end{array}$ \\
\hline
\end{tabular}

\section{Classification results after attributes reduction}

For some cases, not all attributes are necessarily correlated with the final classification of the SVM. Mathematically, there exists a minimal set of attributes which can achieve the optimal decision boundary. The Weka toolbox provides a "featureselection" facility based on the evaluation of the classification effect of each attribute and the correlations among them. Thus a smaller attribute set can be obtained by feature selection: $\left\{V_{c m i n}, I_{a m i n}, I_{b m i n}\right\}$. By 10 -fold cross validation test, the classification accuracy is $97.6271 \%$, which satisfies the realistic industrial needs.

\section{E. Comparison among different kernels}

To visualize the effects of different kernels (Gaussian, linear and quadratic) on the decision surface, three groups of 3D plots are presented in Fig. 6, Fig. 7 and Fig. 8. Each point represents a system state.

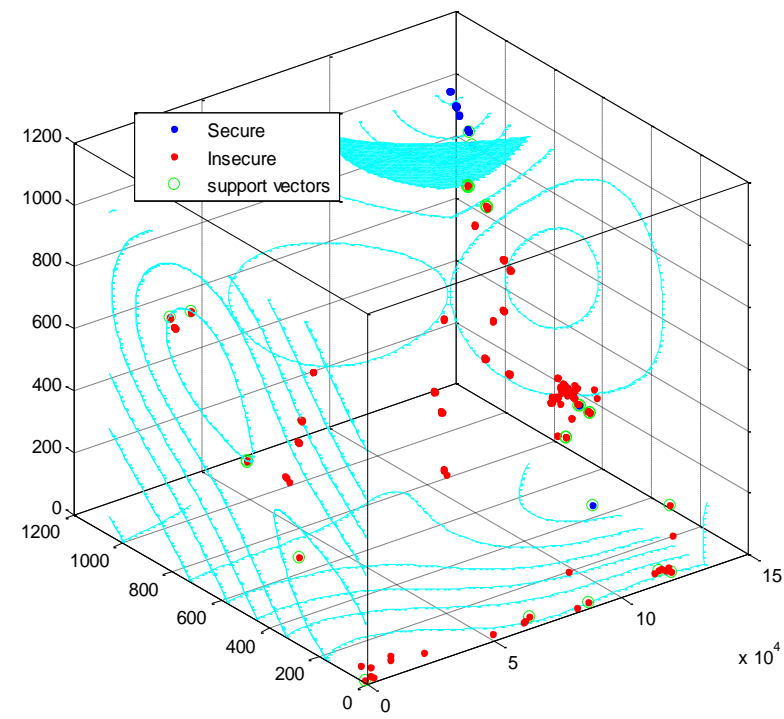

Figure 6. SVM decision surface (Gaussian kernel), accuracy: $99.322 \%$

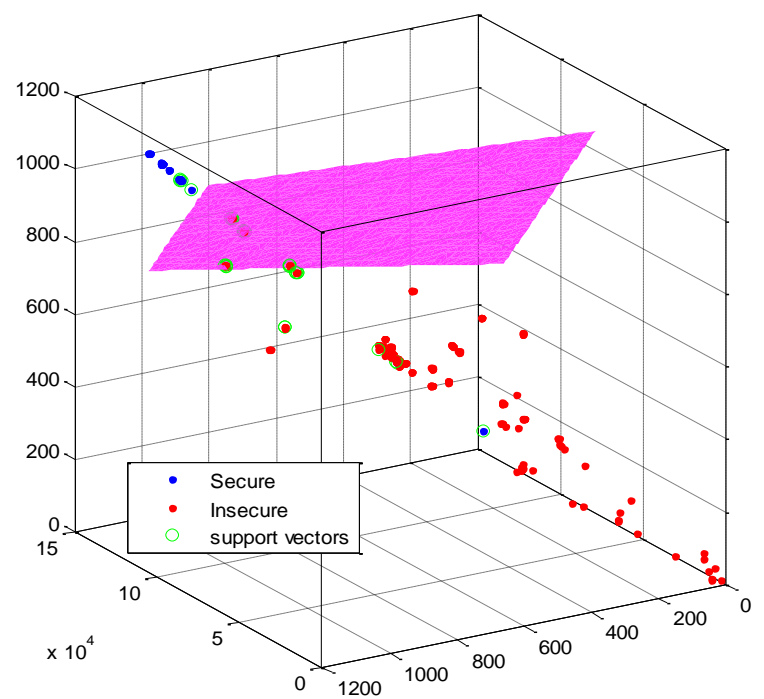

Figure 7. SVM decision surface (linear kernel), accuracy: $99.322 \%$

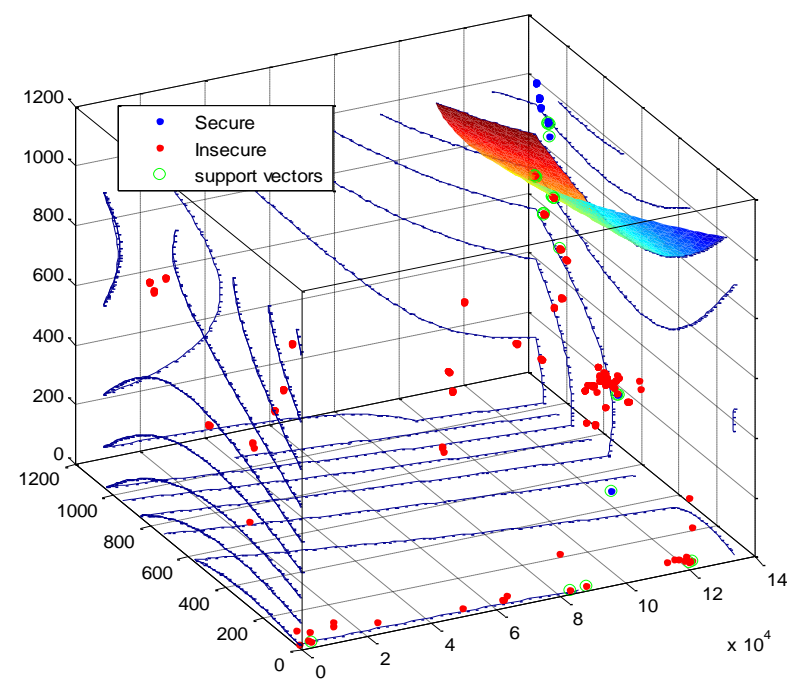

Figure 8. SVM decision surface (quadratic kernel), accuracy: $99.322 \%$ 
From the above plots, it can be observed that:

1) Nonlinear kernels (cases 1 and 3) have a better general ability than a linear kernel. Graphically, their separation surfaces are more flexible than a linear plane;

2) No kernel is $100 \%$ accurate and each has a small number of misclassified cases;

3) For the islanding decision purpose in this paper, a linear kernel is good enough and has less computational complexity.

\section{F. Comparison with Decision trees}

The performance comparison results of the SVM and DT are shown in Table 6, where the accuracies of the SVM and DT are respectively in the left column and right column under each of the three testing cases with three testing methods:
1) Testing on the whole training set;

2) 10-fold cross validation

3) Randomly selected $1 / 3$ of the dataset for testing.

Eight group experiments are compared. Each experiment has a different combination of attributes. From the comparisons,

1) The accuracy of the SVM is close to that of the DT.

2) The SVM sacrifices accuracy of training stage compared to the DT. However, this can help reduce the "over-fitting" drawback with the DT.

3 ) In the cases with only one input attribute, the SVM is not as accurate as the DT. In other words, the SVM may require enough attributes to make a correct decision. Thus how to incorporate a DT's merits into the SVM to enhance its performance in microgrid security assessment may be studied.

TABLE VI. SVM (LEFT-COLUMN) VERSUS DT (RIGHT COLUMN)

\begin{tabular}{|c|c|c|c|c|c|c|}
\hline Attribute & \multicolumn{2}{|c|}{$\begin{array}{c}\text { Accuracy } \\
\text { [Training only] }\end{array}$} & \multicolumn{2}{|c|}{$\begin{array}{c}\text { Accuracy } \\
\text { [Cross Validation, } 10 \text { Folds] }\end{array}$} & \multicolumn{2}{|c|}{$\begin{array}{c}\text { Accuracy } \\
\text { [Training: 66\%; Testing: } \\
34 \% \text { ] }\end{array}$} \\
\hline Iamax, Icmin & 97.9661 & 98.9831 & 97.9661 & 98.3051 & 99 & 99 \\
\hline Vbmax, Qmax & 97.6271 & 98.9831 & 97.6271 & 97.9661 & 99 & 99 \\
\hline Qmin, Pmin & 98.9831 & 98.9831 & 98.9831 & 98.3051 & 100 & 100 \\
\hline Vcmax, Vbmin & 98.3051 & 98.9831 & 98.3051 & 98.6441 & 99 & 100 \\
\hline Pmax, Iamax & 98.9831 & 98.9831 & 98.9831 & 98.3051 & 99 & 99 \\
\hline Vbmin, Qmin & 98.9831 & 98.9831 & 98.9831 & 98.6441 & 100 & 100 \\
\hline Vamax & 94.2373 & 98.9831 & 94.2373 & 98.6441 & 92 & 99 \\
\hline Ibmax & 95.9322 & 98.9831 & 78.9831 & 98.3051 & 73 & 99 \\
\hline
\end{tabular}

\section{CONCLUSIONS}

This paper proposed a SVM-based method for microgrid islanding decision and control. The method was tested on a microgrid model integrating three different types of DGs and demonstrated a high accuracy in classifying cases with or without the need of islanding control by only using measurements from the PCC bus of the microgrid. The SVM used in the method was also compared to the DT.

\section{REFERENCES}

[1] M. Dewadasa, A. Ghosh and G. Ledwich, "Protection of Microgrids Using Differential Relays," 21st Australasian Universities Power Engineering Conference (AUPEC), 2011.

[2] F. Katiraei, M. R. Iravani and P. W. Lehn, "Microgrid Autonomous Operation During and Subsequent to Islanding Process," IEEE Trans. Power Delivery, Vol.20, No.1, pp. 248-257, Jan. 2005.

[3] K. Sun, S. Likhate, V. Vittal, S. Kolluri, S. Mandal, “An Online Dynamic Security Assessment Scheme Using Phasor Measurements and Decision Trees," IEEE Trans. Power Syst., vol. 22, pp. 1935-1943, Oct. 2007.

[4] R. Diao, K. Sun, V. Vittal, R. J. O'Keefe, M. R. Richardson, N. Bhatt, D. Stradford, S. K. Sarawgi, "Decision Tree-Based Online Voltage Security Assessment Using PMU Measurements," IEEE Trans. Power Systems, vol. 24, pp.832-839, May 2009.

[5] C. Liu, K. Sun, Z.H. Rather, Z. Chen, C. L. Bak, P. Thøgersen, P. Lund, "A Systematic Approach for Dynamic Security Assessment and the Corresponding Preventive Control Scheme Based on Decision Trees," IEEE Trans. Power Syst., vol. 29, no. 2, March 2014.

[6] E. Bernabeu, J. Thorp and V. Centeno, "Methodology for a Security/Dependability Adaptive Protection Scheme Based on Data Mining," IEEE Trans. Power Delivery, Vol. 27, pp.104-111, Oct. 2012.

[7] R. Azim, Y. Zhu, H. A. Saleem, K. Sun, F. Li, D. Shi, R. Sharma, "A Decision Tree Based Approach for Microgrid Islanding Detection", IEEE Innovative Smart Grid Technologies (ISGT) Conference, 2015
[8] S. kalyani and K. S. Swarup, "Classification and Assessment of Power System Security Using Multiclass SVM," IEEE Trans. Syst. Man Cy. C, Vol. 41, No. 5, Sept. 2011.

[9] M. He, V. Vittal and J. Zhang, "Online Dynamic Security Assessment with Missing PMU Measurements: a Data Mining Approach," IEEE Trans. Power Syst., vol. 28, pp. 1969-1977, May. 2013.

[10] F. R. Gomez., A. D. Rajapakse., U. D. Annakkage, and I. T. Fernando, "Support Vector Machine Based Algorithm for Post-Fault Transient Stability Status Prediction Using Synchronized Measurements," IEEE Trans. Power Syst., vol. 26, pp. 1474-1483, Nov. 2011.

[11] K. Seethalekshmi, S. N. Singh and S. C. Srivastava, "A Classification Approach Using Support Vector Machines to Prevent Distance Relay Maloperation Under Power Swing and Voltage Instability," IEEE Trans. Power Delivery, vol.27, no.3, pp. 1124 - 1133, June 2012.

[12] W. H. Kersting. Radial distribution test feeders. [Online]. Available: http://ewh.ieee.org/soc/pes/dsacom/testfeeders/

[13] Chih-Chung Chang and Chih-Jen Lin. LIBSVM: A Library for Support Vector Machines. ACM Transactions on Intelligent Systems and Technology, vol. 2, pp. 27:1--27:27, 2011. [Online]. Available: http://www.csie.ntu.edu.tw/ cjlin/libsvm.

[14] Remco R. Bouckaert, Eibe Frank, Mark Hall, et al. Weka menu for vers ion 3-6-10. July 31, 2013. [Online]. Available: http://www.cs.waikato.a c.nz/ml/weka/docume-ntation.html. 